\title{
IOT INTEGRATED FOREST FIRE DETECTION AND PREDICTION USING NODE MCU
}

\author{
M. Khadar, V. Ranjith, K. Varalakshmi
}

\begin{abstract}
Forest fires in Riau Province Indonesia are issues that regularly happen with affected the length and breadth of Indonesia. The effects forest fire and of haze on human health as reported in that particular year were about 20 million people have suffered from respiratory problems and serious deterioration in overall health in Riau community. This research proposes development of Wireless Sensor Network (WSN) for detection of forest fire in Riau province for the region that high risk forest fire in dry session, with WSN technology data be able to collect from the sensor deploy in the forest area. The deployment of sensors will be located at several locations that has badly impacted in previous case and forecast location with potential fire happen. Mathematical analysis is used in this case for modelling number of sensor required to deploy and the size of forest area represented overall of Riau Province. An early indication of forest fires is needed for quick prevention before they become uncontrollable and overwhelming. The design and development of WSN sensors give high feasibility to overcome current issues in Riau Province because of forest fire. The development of this system used WSN highly applicable for early warning and alert to representative institution for action taken.
\end{abstract}

Index Terms WSNs, Forest Fire, Sensors, Detection

Reference to this paper should be made as follows:

M. Khadar, V. Ranjith, K Varalakshmi, "Iot Integrated Forest Fire Detection and Prediction using NodeMCU” Int. J. of Electronics Engineering and Applications, Vol. 9, No. 1, pp. 28-35, doi 10.30696/IJEEA.IX.I.2021.28-35. 


\section{[I] INTRODUCTION}

Indonesian suffer from badly haze due to land and forest fires that happen almost every year. The location of Indonesia at equatorial causes this country to have longer dry season spans from April to October. Riau province is one of the state that has high threat to land and forest fire due to peatland, particularly in industrial forest areas. Most of the fires occurring in peat forests are serious due to the characteristics of peat which is easily flamed due to continuous dry season. It has been reported that the total economic loss for Riau province in year 2015 due to this disaster was about USD1.65 billion. More worst when it has huge impact on local environment, flora, fauna and human health. Elderly people and children are severely affected due to haze. Furthermore, the impact of this land forest fire is not only in Indonesia or Riau Province but also has caused deterioration in air quality and human health problem in others countries like Malaysia and Singapore. Current detection method is using satellite to detect any hotspot of land and forest fires. Such data however may not sufficient as the satellite cannot provide fine hotspots detection at other potential areas. The local authorities are normally depending on the satellite imagery to make a decision or report from local community and company.

Therefore, in this research focus on developing ground level smart monitoring system to detect and monitor the environmental behavior in term of temperature, humidity and gasses. Proposing a new technology for monitoring system using low power wireless data communication with LoRa- IoT technology. The integration of sensors with LoRa technology would have an effect to local community where people could access the information through developed real- time database in anytime. This ground level detection method will be deployed in other areas, regions and states in Indonesia. It is anticipated to be quicker and cheaper solution than to satellite data acquisition and this would definitely be beneficial to social welfare and economy development. In addition, the development of real-time database would also require some support from them as a policy maker to understand how the system works and also understand the pattern of the results so that an appropriate action can be taken.

\section{[2] RELATED WORK}

Wireless Sensor Networks (WSNs) can be used for many applications, such as in remote environmental monitoring, industrial automatic control, remote sensing and target tracking. The similar application system is in environmental monitoring system which is for forest fires detection that can make a real-time monitoring and detection. Wireless sensor network consists of many and numerous number of small nodes in most situations, which small nodes are deployed in remote and inaccessible hostile environments or over large geographical areas. The large number of sensor small nodes sense environmental changes and report them to cluster head node or sensor base station, then through a gate way to transfer data to the servers which the deployment and maintenance should be easy and scalable.

Several research on Wireless Sensor Network (WSN) as discuss in [1], the WSN Simulator is developed based on proposed Sensor model and WSN model. The WSN Simulator address important design issues as: coverage of the area under surveillance in relation to initial sensor deployment, number of sensors needed for targeted deployment, and coverage change as function of time. A new approach for forest fire monitoring and detection as discussed in [2] which using data aggregation in WSN. The proposed approach can provide faster and efficiently reaction to forest fires while consuming economically WSN's energy, which has been validated and evaluated in extensive 


\section{Khadar et al.}

simulation experiments. Wireless sensor network be able to provide better solution for disaster management and rescue operations such as earthquake detection and alert system, flood detection, landslide detection, forest fire detection, water level monitoring of Himalayan Rivers, monitoring of glaciers, pilgrimage and tourist management are various examples where WSN can be used. Sensors are deployed for measuring various parameters and on [3, 4].

WSN algorithm to identify malicious data injections and build measurement estimates that are resistant to several compromised sensors and even when they collude in the attack. The methodology to apply this algorithm is in different contexts and evaluates its results on three different datasets drawn from distinct WSN deployments $[5,6]$. The others research have been done is application of WSN in predicting natural disasters like hailstorm, fire, rainfall etc. by WSN are infrequent and stochastic [7]. As well as in design and implementation of a smart fire detection system using a WSN and Global System for Mobile (GSM) communication to detect fires effectively and reduce false positives, the system uses smoke and temperature sensors [8,9]. Application of WSN in energy conservation, reducing data transmission delay and improving the network lifetime. Used of clusterchain mobile agent routing (CCMAR) for low energy adaptive clustering hierarchy (LEACH) and power- efficient gathering in sensor information systems (PEGASIS) [10-13].

this case network is stationary, without mobile WSN sensors, but sensor positions are time dependent, since sensor nodes are expected to stop operating in time. In this cease operation can have different causes: hardware faults, accidental, battery depletion, and intentional sensor removal, etc.

$$
I P=\frac{\text { area covered with sensors }}{\text { the total area of the surveillance region }} \cdot 100 \%
$$

The basic model component is a WSN sensor node defined as a vector:

$$
S=(d, E(t))
$$

where $\mathrm{d}$ is a range of sensor transmission, or radius of transmission area, the area covered by radio signal for data exchange with a neighboring node. $\mathrm{E}(\mathrm{t})$ is energy available for sensor power supply. Assume a homogenous sensor network with $\mathrm{n}$ unified type sensors and one hub-sensor for communication with a dispatcher node. Network parameters are described as a vector:

\section{[3] WSN IN FOREST FIRE DETECTION ANALYSIS}

Number of fire hotspot based on satellite imaginary monitored in Riau Province scattered in most of area especially in southern region. Fig. 1 shows number of hotspots, based of pattern scattered distribute over in all of district in Riau Province. 


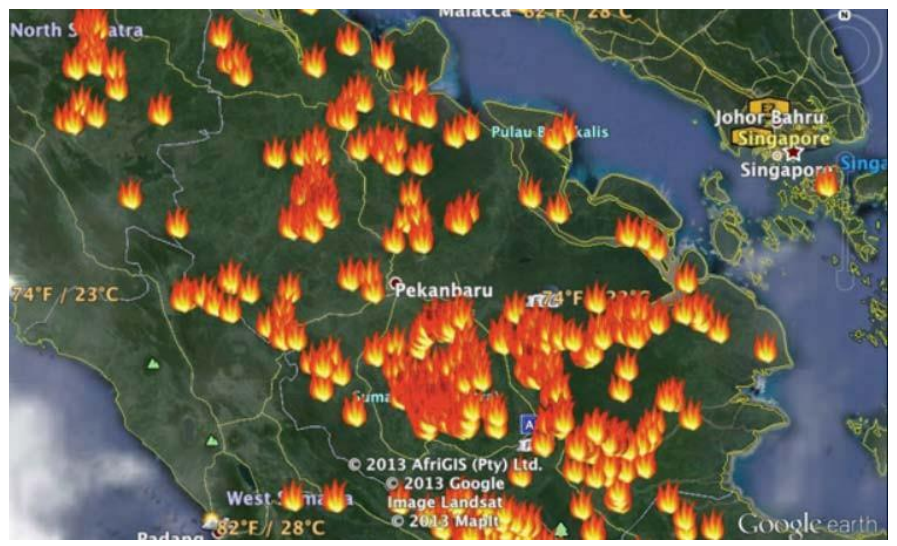

Fig. 1. Hotspot in Riau Province based on satellite image.

Area of hotspot coverage assumes a set of WSN sensors distributed over a geographical region in Riau Province to monitor that area. Coverage function $\mathrm{P}$ is given as:

$$
\begin{aligned}
& P=f(x, y, t)=\left\{\left(x_{1}, y_{1}\right), \ldots\left(x_{n}, y_{n}\right)\right\}, \\
& \left(x_{k}, y_{k}\right)=f(t), k=1,2,3, \ldots, n
\end{aligned}
$$

where $(\mathrm{x}, \mathrm{y})$ are coordinates of sensor within the monitored region, and $\mathrm{t}$ is time. Model is using a projection in the $2 \mathrm{D}$ space of a fire surveillance region, which is a $3 \mathrm{D}$ sphere. In

$M=\left(n, f_{0}, \Delta E\right)$

where $\mathrm{n}$ is the number of sensors, for is the frequency of regular transmissions, and OE is energy consumption per transmission. Assume that sensor nodes periodically transmit the data collected to the neighboring nodes. Energy consumption OE includes also energy spent in sensing and data processing. Each node has two roles:

(a) sensing environmental data and its transmission.

(b) receiving data from neighboring nodes and forwarding.

The sensing role is defined in accordance with the WSN sensor network application, and can be easily influenced with sensor node type selection. Energy consumption OE is thus linked to the sensor node type and its value is listed in the sensor node data sheet. The forwarding neighboring sensor node data role is primary defined by communication protocol. WSN simulator having knowledge of sensor nodes positions and defines paths for data forwarding employing optimization algorithms. Assume that routing optimization would be implemented in the real protocol as well, but we do not address that issue in our work. In order to simplify the model, each sensor node is aware of its GPS co-ordinates, which are used in communication as an identification code. It is also assumed that the hub node initially broadcasted across all the nodes. Based on these assumptions it is possible to 


\section{Khadar et al.}

implement optimized routing algorithm. Energy consumption needed for receiving and forwarding neighbor data is $\mathrm{OE}$.

Object under surveillance is modeled as four-side stationary polygon defined as a set:

$O=(A, B, C, D)=\left\{\left(x_{A}, y_{A}\right),\left(x_{B}, y_{B}\right),\left(x_{C}, y_{C}\right),\left(x_{D}, y_{D}\right)\right\}$

where $\mathrm{A}, \mathrm{B}, \mathrm{C}$ and $\mathrm{D}$ are polygon points with co-ordinates ( $\mathrm{x}, \mathrm{y})$.

The role of WSN hub sensor node is to collect data from each sensor node and forward the data to base station or co- ordination center. Data package received and forwarded by the hub node contains originator sensor node address and measurement values (temperature, humidity and $\mathrm{CO} 2$ ). The WSN hub node has uninterrupted power supply and that communication channel between the hub node and co- ordination center is unremitting. Hence, simulation is treating the hub sensor as "constantly available". The main objective of the simulation is to optimize network routes for data transmission from sensor nodes to the hub node [1].

\section{[4] DEVEOPMENT WSN IN FOREST FIRE DETECTION}

Forest fires are a natural and recurrent phenomenon or manmade, in many case of the world. Burning areas are mainly located in temperature climates where its rainfall is high enough to enable a significant level of vegetation, but in summers session are very hot and dry environment, be able to create a dangerous fuel load. Global warming will contribute to increase the number and importance of these disasters. In every season, not only are thousands of forest hectares destroyed by wild land fires, but also properties, assets and public resources and facilities are destroyed because of fire. Moreover, firefighter and civilians are at risk, to handle with a terrible toll in human lives each year. Fig. 2 shows diagram of a numbers of WSN sensor deploy in forest area for fire detection.

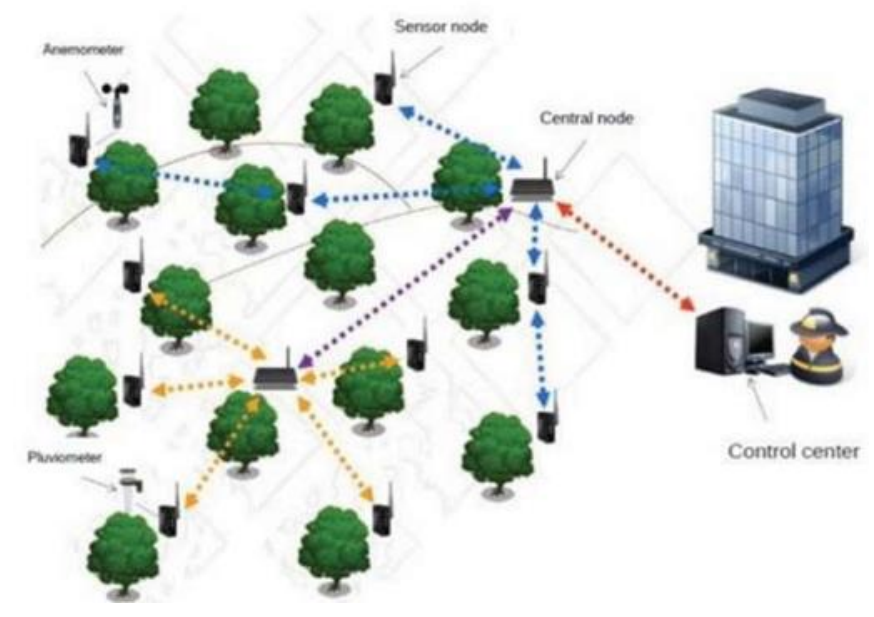

Fig. 2. Topology of WSN sensor nodes deploy in forest for fire detection.

A forest fire in general a dynamic phenomenon that may changes its properties and behavior by the time from one place to another and with the passage of time. In the fact that the forest fuel available in a given location is limited, for a fire to continue it must spread to neighboring fuel. This is performed through the complex heat spread to neighboring fuel and performed through the complex fire behavior. Another approach is also based on the WSN paradigm has been designed and developed in the context of a research project that included all the key actors in forest as well as fire fighting for operations. This unique proposed ecosystem has provided the solution with a holistic 
perspective that results in a set of distinguishing features, which all node types can include environment and meteorological sensors.

Another scenario is in fig. 3 shows a schematically structure proposed of the development ZigBeeWSN-based system for forest fire detection and protection management, consisting of multi-sensor nodes, coordinators, cluster heads, routers and remote decision server. This cluster-tree network topology structure proposes design to reduce the loss of energy and data package while transferring. ZigBee technique is a global standard based on IEEE 802.15.4 applicable for low-rate wireless Personal Area Networks (PAN).

ZigBee is one of the wireless network standard targeted at low power sensor that apply in multi frequencies $868 / 915 \mathrm{MHz}$ and $2.4 \mathrm{GHz}$. The technical advantage proposes of ZigBee is to offer a system with long battery life, small size, low-cost, high reliability and automatic or semi- automatic installation.

Therefore in this development design WSN node to achieve an optimal choice for forest fire detection and monitoring [11].

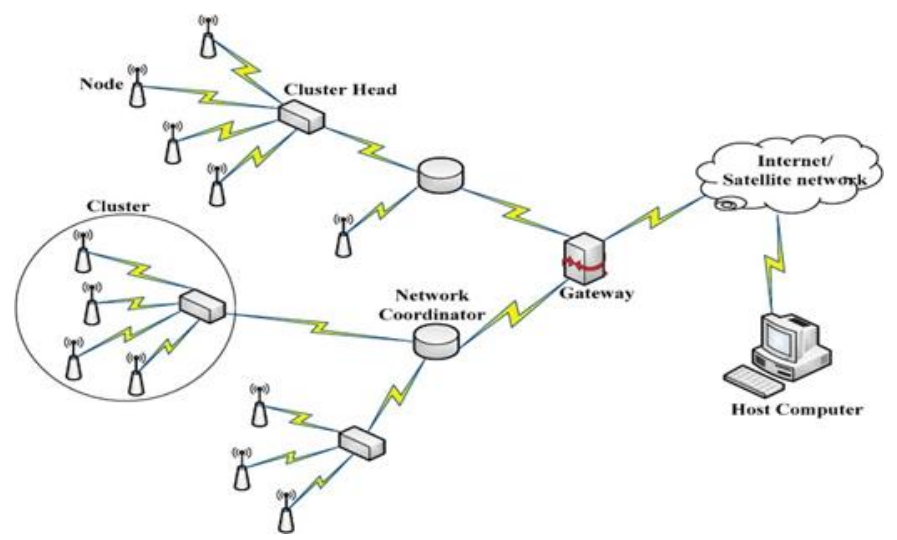

Fig.3. A WSN sensor nodes use ZigBee standard.

Actual hardware on WSN node for fire detection and monitoring can be found in many types in the market. Where temperature, humidity, smoke and carbon sensor installed in the node to detect all the parameter that high relation to the forest fire. Figure 4 shows actual fabricated sensor ready to deploy, before sensor node deploy in the field the sensor nodes have to configure based on design and requirement. All the node will send a data or message to the WSN coordinator that has function to receive all information from node scattered.

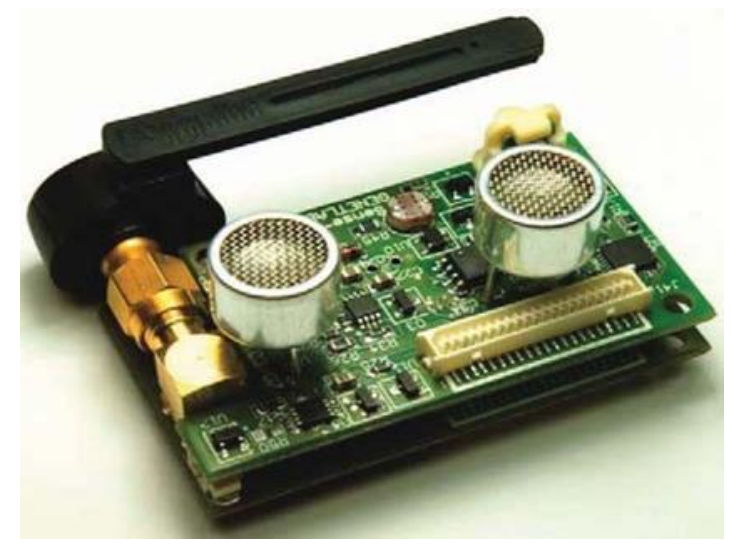




\section{Khadar et al.}

Fig.4. Actual of WSN nodes with sensors for fire detection

\section{[5] CONCLUSION}

Development of WSN nodes for forest fire detection and furthermore for monitoring have been proposed. In this proposed design analysis use mathematical approach according to the area has to cover which in the whole Riau Province. Temperature, smoke and CO2 sensor are high light in this case because of those parameters are principle parameters to the fire case either in the land or forest. Proposed design sensors node use ZigBee model, with low power then sensor nodes can use in long life as node powered by battery. In order to cover the whole of Riau province, minimum have to create network coordinator in each of Kecamatan and one gate to access in server (cloud database) as well monitoring computer. Theoretical proposed concept of WSN very applicable to use for detection forest fire, especially in Riau Province case. 


\section{REFERENCES}

[1]. A. Aksamovic, M. Hebibovic, and D. Boskovic, "Forest fire early detection system design utilizing the WSN simulator," in 2017 XXVI International Conference on Information, Communication and Automation Technologies (ICAT), 2017, pp. 1-5.

[2]. Y. Liu, Y. Liu, H. Xu, and K. L. Teo, "Forest fire monitoring, detection and decision making systems by wireless sensor network," in 2018 Chinese Control And Decision Conference (CCDC), 2018, pp. 54825486.

[3]. D. Pant, S. Verma, and P. Dhuliya, "A study on disaster detection and management using WSN in Himalayan region of Uttarakhand," in 2017 3rd International Conference on Advances in Computing, Communication \& Automation (ICACCA) (Fall), 2017, pp. 1-6.

[4]. C. Aranzazu-Suescun and M. Cardei, "Distributed algorithms for event reporting in mobile-sink WSNs for Internet of Things," Tsinghua Science and Technology, vol. 22, no. 4, pp. 413-426, 2017.

[5]. V. P. Illiano and E. C. Lupu, "Detecting Malicious Data Injections in Event Detection Wireless Sensor Networks," IEEE Transactions on Network and Service Management, vol. 12, no. 3, pp. 496-510, 2015.

[6]. E. A. Kadir, S. L. Rosa, and H. Gunawan, "Application of RFID technology and e-seal in container terminal process," in 2016 4th International Conference on Information and Communication Technology (ICoICT), 2016, pp. 1-6.

[7]. A. Kansal, Y. Singh, N. Kumar, and V. Mohindru, "Detection of forest fires using machine learning technique: A perspective," in 2015 Third International Conference on Image Information Processing (ICIIP), 2015, pp. 241-245.

[8]. M. Saoudi, A. Bounceur, R. Euler, T. Kechadi, and A. Cuzzocrea, "Energy-Efficient Data Mining Techniques for Emergency Detection in Wireless Sensor Networks," in 2016 Intl IEEE Conferences on Ubiquitous Intelligence \& Computing, Advanced and Trusted Computing, Scalable Computing and Communications, Cloud and Big Data Computing, Internet of People, and Smart World Congress (UIC/ATC/ScalCom/CBDCom/IoP/SmartWorld), 2016, pp. 766-771.

[9]. S. Sasirekha and S. Swamynathan, "Cluster-chain mobile agent routing algorithm for efficient data aggregation in wireless sensor network," Journal of Communications and Networks, vol. 19, no. 4, pp. 392-401, 2017.

[10]. E. A. Kadir, "A reconfigurable MIMO antenna system for wireless communications," in 2017 4th International Conference on Electrical Engineering, Computer Science and Informatics (EECSI), 2017, pp. $1-4$.

[11] Abu Salim, Sachin Tripathi and Rajesh Kumar Tiwari "A secure and timestamp-based communication scheme for cloud environment" Published in International Journal of Electronic Security and Digital Forensics, Volume 6, Issue 4, 319-332.

[12] Rajesh Kumar Tiwari and G. Sahoo, "A Novel Watermark Scheme for Secure Relational Databases" Information Security Journal: A Global Perspective, Volume 22, Issue 3, July 2013.

[13] Anoop Joyti Sahoo, and Rajesh Kumar Tiwari "A Novel Approach for Hiding Secret data in Program Files” International Journal of Information and Computer Security. Volume 8 Issue 1, March 2016, 